\title{
Numerical Prediction of NPSHR Characteristics of Double Suction Centrifugal Pump
}

\author{
Shyam Narayan Shukla ${ }^{\# 1}$, Ruchi Khare ${ }^{* 2}$, Vishnu Prasad ${ }^{* 3}$ \\ \# Kirloskar Brothers Limited, Pune, India, 411045 \\ ${ }^{1}$ Shyam.shukla@kbl.co.in \\ * Maulana Azad National Institute of Technology, \\ Bhopal, India, 462003 \\ ${ }^{2}$ ruchif1@yahoo.com \\ 3vpp7@yahoo.com
}

\begin{abstract}
The pressure at inlet of pump is mostly below atmosphere pressure and may come below the vapour pressure of water depending on installation and operating condition. This situation may lead to cavitation in pump and it is one of the leading causes for reduced reliability on performance of centrifugal pumps. NPSH (net positive suction head) is used to check the cavitation condition in centrifugal pumps and it is the absolute total pressure energy available above vapour pressure. The NPSHR is the net positive suction head required at pump inlet for working of pump without cavitation and special test rig is required to predict the values of NPSHR of pump experimentally. CFD has minimized the experimental work for prediction of cavitation characteristics of hydraulic machines where selection of turbulence model plays important role on the accuracy of simulation results. In this paper, an effort has been made to predict NPSHR characteristics of double suction centrifugal pump at three different operating points using Computational Fluid Dynamics (CFD) approach with two turbulence models. The results from CFD using $\kappa-\varepsilon$ and SST turbulence models have been compared with experimental results at pump design condition and found to be in close comparison. The losses in different pump components are also computed to study pattern of hydraulic loss with the growth of cavitation.
\end{abstract}

Keyword- Centrifugal pump, cavitation, Net positive suction head, CFD analysis, Double suction.

\section{INTRODUCTION}

Water flows from high energy to low energy level. The rotation of pump impeller creates low pressure at the inlet and due to this; water in sump at the atmospheric pressure is sucked into the pump. The difference between suction pressure (stagnation) and vapour pressure is Net positive suction head (NPSH). It is the energy of liquid required to overcome the friction losses from the suction nozzle to the eye of the impeller without causing vaporization. Centrifugal pumps must have minimum NPSH to function properly and it is known as net positive suction head required (NPSHR) and is specified by the pump manufacturer. The net positive suction head available to pump (from the system to which the pump is attached), is designated as the net positive suction head available (NPSHA). NPSHA is dependent on site installation and operating conditions of pump in pumping system [1]. NPSHA must be greater than NPSHR for the pump system to operate without cavitation. When the total absolute pressure at inlet of the pump is less than the water vapour pressure, then water starts to vaporize and boil. The vapour bubbles formed near impeller inlet continue to move through the impeller and collapse in areas of higher pressure within the pump [2]. The frequent collapse of bubbles develops extremely high localized pressure and blows small portions of metal from the impeller surface.

When cavitation occurs in a pump, its efficiency reduces and can also cause surges in flow. When the volume of water is subjected to a sufficiently low pressure, it may change its phase from water to gaseous resulting in vapour bubbles[3]. The process of formation of vapour bubble in a space and their subsequent collapse in high pressure space is referred as cavitation. If the pump operates under cavitation conditions for enough time, it createsa loud noise and vibration, pitting marks on the impeller blades and volute casing wall, premature seal and bearing failure and other fatigue failures in the pump[4].

The reduction of pressure at pump inlet may also be caused due to increase of the temperature of water and reduction of the flow area by obstructions or sharp curvatures in piping or pump. 
The NPSHR characteristic of the pump is indicated on the pump's curve. According to the standards of the Hydraulic Institute, a suction lift test is performed on the pump and the pressure in the suction vessel is lowered to the point where the pump suffers a $3 \%$ head drop. The NPSH at point is called the NPSHR (Net positive suction head required) of the pump. The experimental approach for prediction of cavitation needs a wellequipped laboratory and it is tedious and time consuming. The prediction of cavitation in pumps using CFD is cost and time effective but needs proper selection of simulation parameters like turbulence models for good accuracy[5].

The present paper deals with numerical prediction of NPSHR characteristic of double suction centrifugal pump at three different operating points (i.e. $80 \%, 100 \%$ and $120 \%$ of duty point). The numerical flow simulation is carried out using two turbulence models ( $\kappa-\varepsilon$ and SST). An effort hasalso been made to assess the hydraulic loss pattern in the pump due to cavitation in different components. The numerically predicted NPSHRcharacteristic at duty point is compared with experimental results and bears close comparison. Commercially available software ANSYS CFX 16.1 is used for numerical flow simulation.

\section{GEOMETRY FOR FLOW SimULATION}

The pump under investigation is a double suction horizontally split casing pump. It consists of stationary suction passage divided into two halves, rotating double suctionfive vane impeller and a stationary volute casing. The pump design head coefficient and discharge coefficient are 0.433 and 0.223 respectively with specific speed NSUS 1710. A solid model of the entire assembly has been prepared using solid modeller package Pro-Engineer as shown in Fig. 1. The front and isometric views of double suction impeller are shown in Fig. 2.

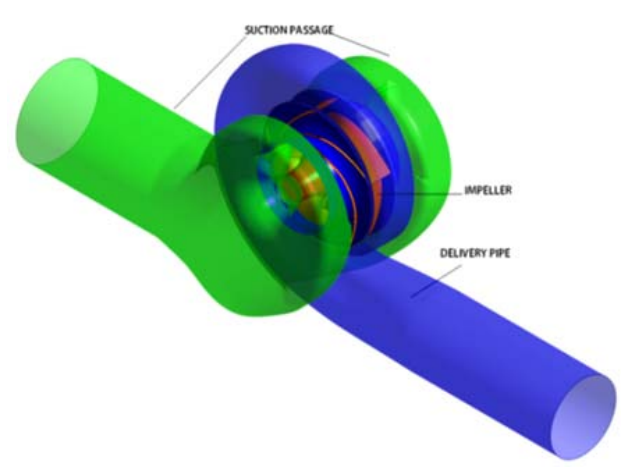

Fig.1. Solid model of pump assembly

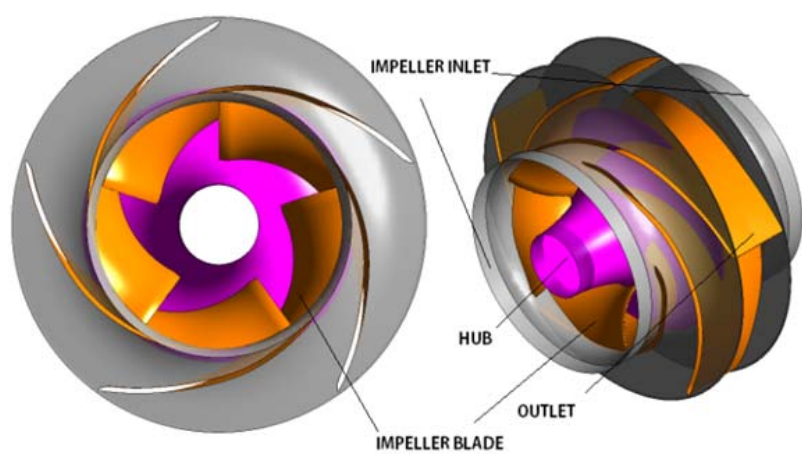

Fig. 2. Front and isometric view of impeller

The assembly of pump parts imported to ANSYS ICEM CFD16.1 for geometry cleaning and meshing. The unstructured hybrid tetrahedral mesh is generated in flow domain except fine prismatic layers near the surface of impeller vanes for proper resolution of the boundary layer. Grid independence study has been performed to ensure appropriateness of grid size and Y+ value. There are nearly 300000, 1120000 and 200000 nodes in suction passage, impeller and delivery casing respectively.

Eight turbulence models had been chosen for numerical flow simulation [6] but $\kappa-\varepsilon$ and SST models were found to give comparable results with the experimental ones. Hence these two models have been chosen for cavitation analysis. The flow simulation for cavitation has been carried out in two stages i.e. single phase and multiphase flow. Initially, the performance analysis of the pump was carried out using single phase flow to generate initial guess for cavitation analysis.

Multiphase flow analysis was carried out using Eulerian approach option available in ANSYS CFX 16.1 software.In single phase analysis, the mass flow rate was specified at the outlet of delivery casing. Impeller rotation was specified to impeller domain and assigned as rotating domain[7]. Suction passage and delivery passage were specified as stationary domain. At walls, no slip condition has been imposed. Static pressure at inlet was specified as one atmospheric by considering reference pressure as absolute zero. These results are used as initial guess to cavitation solution. In cavitation analysis, inlet pressure has been varied to estimate $3 \%$ head drop for NPSHR. The morphology of water vapour was set as dispersed fluid with $0.05 \mathrm{~mm}$ diameter as mean diameter. Standard $\kappa-\varepsilon$ turbulence model/ SST turbulence model with scalable wall function was selected for simulation [8]. High resolution advection scheme was used for solution. In the high resolution scheme, the blend factor values vary based on the local solution field in order to enforce a boundness criterion.

The steady state solution has been performed in the relative frame of reference. In multiphase analysis, the two phases are defined as water and water vapour phase at $25^{\circ} \mathrm{C}$. The buoyancy term and suitable gravity components in $\mathrm{x}, \mathrm{y}$, and $\mathrm{z}$ directions were assigned. The water was set as continuous fluid. Morphology was used to describe the connectivity or distribution of the fluid[9]. The difference in density between phases 
produces the buoyancy force in multiphase flows and hence it is important to correctly set the buoyancy reference density. The density of water is set as buoyancy reference density. Rayleigh Plesset model [10] is based on equations for control of vapour generation and condensationis chosen for analysis with saturation pressure of water as $3600 \mathrm{~Pa}$. These equations describe the growth and collapse of the bubbles in different hydrodynamic and thermodynamic conditions

\section{COMPUTATION OF PARAMETERS}

The numerical simulation gives values of pressure and velocity distribution in flow domain of pump. The following local and global flow and loss parameters are computed in non-dimensional form as:

$\begin{array}{ll}\text { Head developed } & H=\frac{T P_{O}-T P_{I}}{\rho g} \\ \text { Relative loss in suction } & \xi_{S}=\frac{H_{S}}{H} * 100 \\ \text { Relative loss in impeller } & \xi_{I}=\frac{H_{I}}{H} * 100 \\ \text { Relative loss in casing } & \xi_{C}=\frac{H_{C}}{H} * 100 \\ \text { Total head loss } & H_{H L}=H_{S}+H_{I}+H_{C} \\ \text { Net positive suction head } & N P S H=\frac{T P_{I}-P_{\text {wvap }}}{\rho g}\end{array}$

\section{RESULTS AND DISCUSSIONS}

The flow simulation for cavitation prediction is carried out for $80 \%, 100 \%$ and $120 \%$ of design flow rate using $\kappa-\varepsilon$ and SST turbulence models. The inlet static pressure is varied to develop cavitation for each flow rate and head developed by pump, NPSH, losses and efficiency are calculated. The results in quantitative and qualitative form are presented.

\section{A. Quantitative Results}

The process of NPSHR estimation is relative to pump head and 3\% drop in head of the pump is taken as required NPSHR of pump. The CFD results from two turbulence models at 100\% flow rate are compared with experimental results. NPSH v/s head plot from experimental results is shown in Fig. 3.

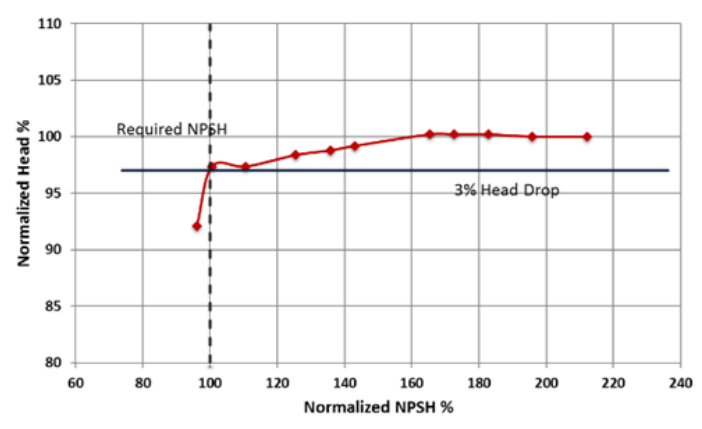

Fig. 3. Plot between pump head and NPSHof experiment results

It is seen from Fig. 3 that as NPSH value reduces, the head developed by pump (normalized w.r.t. pump head without cavitation) is nearly constant till certain value of NPSH and afterwards a small reduction of NPSH leads to reduction in the head ofthe pump. The point where pump head starts reducing is inception point of cavitation. The reduction of pump head continues with further decrease in NPSH. The value of NPSH corresponding to $3 \%$ drop in pump head is the required net positive suction head (NPSHR) of pump.

The pump head and NPSH values obtained from CFD results are normalized with respect to pump head and NPSHR obtained from experimental results. The head variations with NPSH obtained from CFD are shown in Fig. 4 to Fig. 6 for different turbulence condition at $100 \%, 80 \%$ and $120 \%$ of duty point flow respectively. It is observed that pump head is nearly constant with inlet head condition in beginning and then starts falling due 
development of cavitation. In present work, for consistency of NPSH prediction, 3\% drop in pump head at the highest inlet pressure condition is chosen for assessment of NPSHR from CFD results for each flow rate.

It is observed from these figures that trends of pump head variation from both the turbulence models are found to be similar to the experimental results. The value of NPSHR at 100\% flow from SST model in Fig. 4 is very close to experimental value (i.e. $100 \%$ ).

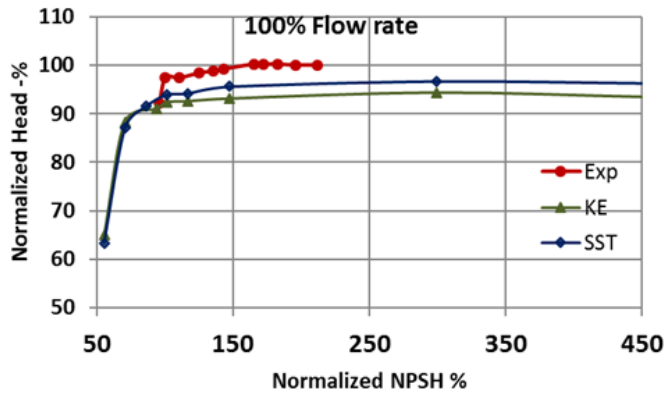

Fig. 4. Head v/s NPSH plot at $100 \%$ flow rate

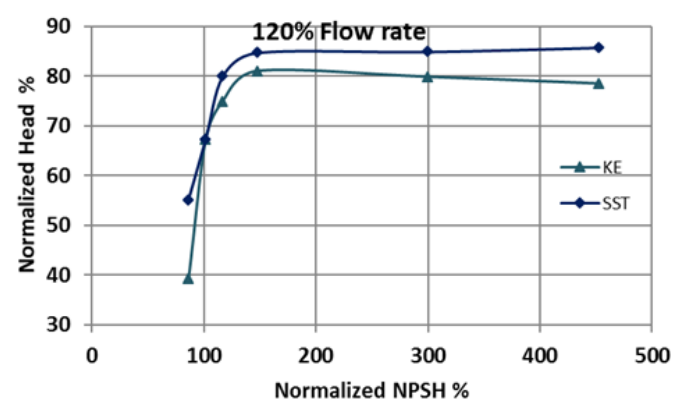

Fig. 6. Head v/s NPSH plot at $120 \%$ flow rate

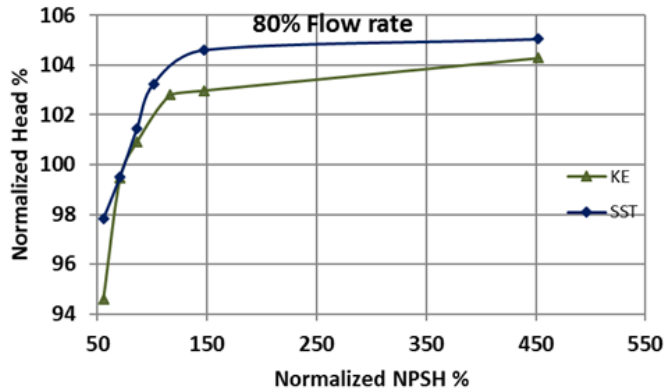

Fig. 5. Head v/s NPSH plot at $80 \%$ flow rate

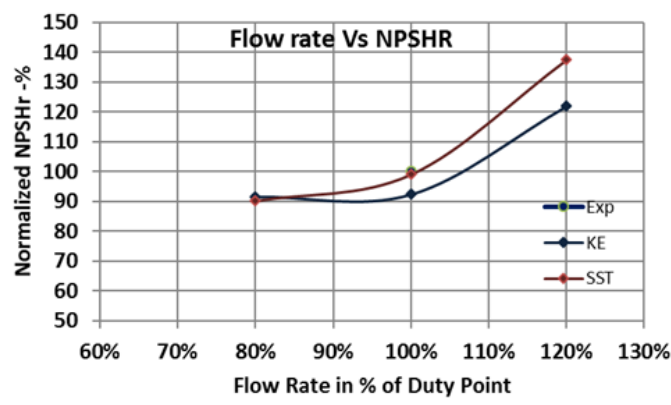

Fig. 7. NPSHR values at different flow rate

TABLE I

Hydraulic Losses in Different Components With and Without Cavitation Conditions

\begin{tabular}{|l|l|l|l|l|l|l|}
\hline \multirow{2}{*}{$\begin{array}{c}\text { Simulation } \\
\text { Condition }\end{array}$} & \multirow{2}{*}{$\begin{array}{c}\text { Flow } \\
(\%)\end{array}$} & \multirow{2}{*}{$\begin{array}{c}\text { Turbulence } \\
\text { model }\end{array}$} & \multicolumn{4}{c|}{ Normalized loss (\%) } \\
\cline { 5 - 7 } & & & HL_Suc & HL_Imp & $\begin{array}{c}\text { HL- } \\
\text { Del_Case }\end{array}$ & HL_Total \\
\hline No_Cav & $80 \%$ & SST & 6.32 & 39.76 & 47.69 & 93.76 \\
\hline No_Cav & $80 \%$ & $\kappa-\varepsilon$ & 6.65 & 40.67 & 52.61 & 99.93 \\
\hline Cav & $80 \%$ & SST & 3.54 & 56.11 & 48.12 & 107.77 \\
\hline Cav & $80 \%$ & $\kappa-\varepsilon$ & 4.15 & 47.73 & 53.48 & 105.36 \\
\hline No_Cav & $100 \%$ & SST & 4.01 & 26.74 & 24.59 & 55.34 \\
\hline No_Cav & $100 \%$ & $\kappa-\varepsilon$ & 6.67 & 33.38 & 31.47 & 71.52 \\
\hline Cav & $100 \%$ & SST & 4.98 & 39.33 & 26.93 & 71.24 \\
\hline Cav & $100 \%$ & $\kappa-\varepsilon$ & 6.03 & 40.86 & 33.38 & 80.27 \\
\hline No_Cav & $120 \%$ & SST & 5.60 & 31.74 & 22.53 & 59.88 \\
\hline No_Cav & $120 \%$ & $\kappa-\varepsilon$ & 7.91 & 37.39 & 33.18 & 78.48 \\
\hline Cav & $120 \%$ & SST & 7.29 & 35.61 & 23.87 & 66.77 \\
\hline Cav & $120 \%$ & $\kappa-\varepsilon$ & 7.40 & 47.14 & 30.98 & 85.53 \\
\hline
\end{tabular}

It is also seen that in CFD simulation for cavitation, the value of pump head and NPSHR from SST model are more than $\kappa-\varepsilon$ model at all operating conditions. 
The hydraulic losses in suction passage, impeller and delivery casing are computed for two turbulence models and three flow rates. The losses in individual component are normalized with the total loss from experimental test for no-cavitation condition and are given in Table I. It is seen that the head losses from SST model are less in comparison to $\kappa-\varepsilon$ turbulence model in all components both in cavitation and non-cavitation conditions. The losses in impeller and delivery casing are significant and increase in cavitation condition while in suction passage; losses are minimum and less than no-cavitation condition (in some cases). It means growths of bubble in such cases are helping to reduce loss. It is also predicted that the numerically computed total loss is less than experimental values except the cavitation condition at $80 \%$ flow.

\section{B. Qualitative Results}

The qualitative results are shown in the form of contour plots of volume fraction of water vapour. As inlet pressure reduces, the amount of water vapour volume fraction increases with reduction of inlet pressure as seen in Fig.8. There is gradual increase initially and later it is much faster and covers nearly complete space at developed stage. The water vapour volume fraction at $1 \mathrm{~atm}$ and $0.4 \mathrm{~atm}$ inlet pressures at $80 \%$ of duty point flow shown in Fig. 9 indicates there is negligible volume fraction zone at latm inlet pressure but slightly more in case of SST model. At inlet pressure of $0.4 \mathrm{~atm}$, it almost covers whole suction side of impeller vane. The generation of water vapour is not symmetric on all the blades. It is also observed that on one vane it covers more area than other vanes. The same trend is seen in the contour plots of different flow rates. The areas covered by water vapour volume fractions are more in SST model at the start of cavitation and less at developed stage as compared to $\kappa-\varepsilon$ turbulence model as seen in Fig. 9 and Fig. 11 at part and over load conditions. At rated conditions in Fig. 10, areas covered by water vapour volume fractions are nearly same in both the turbulence models.

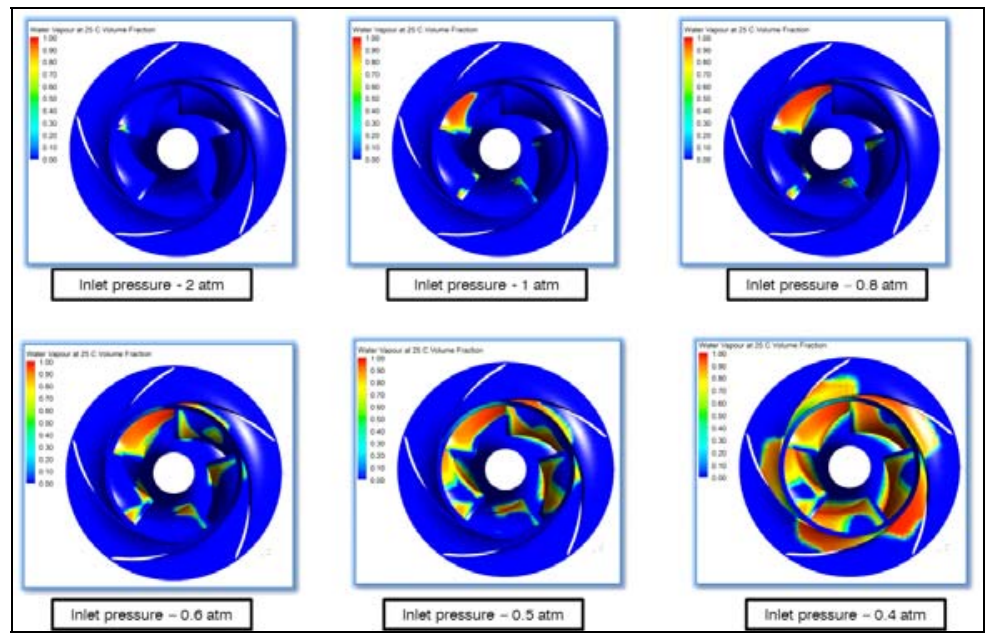

Fig. 8. Water vapour volume fraction development with reduction of pressure at inlet for $100 \%$ flow rate

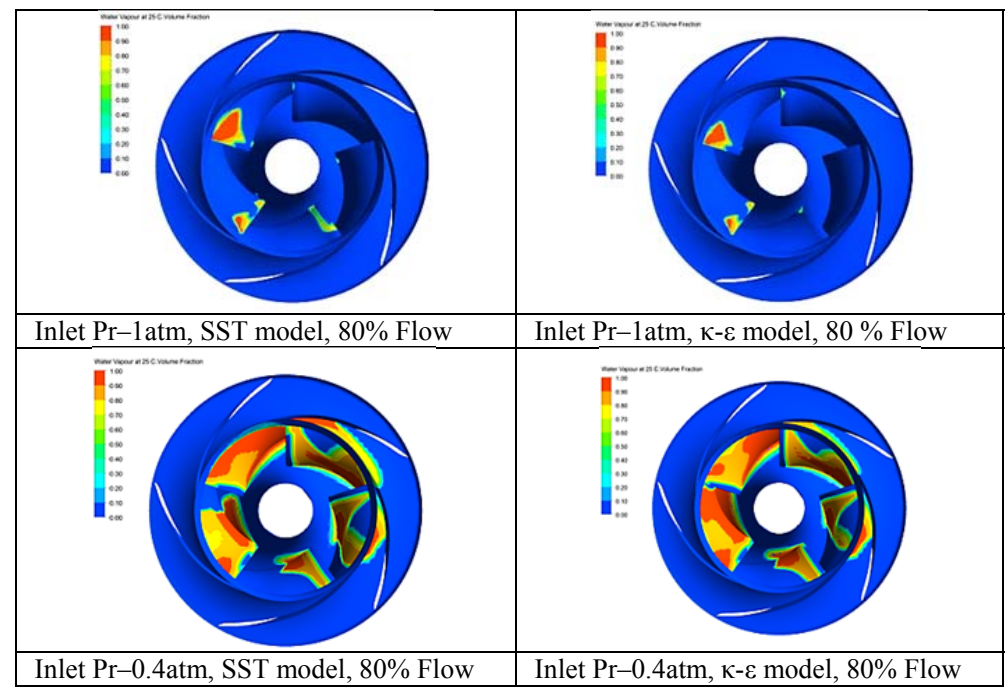

Fig. 9. Comparison of water vapour volume fraction at $80 \%$ of flow rate 
It is seen that the water vapour volume fraction at 1 atm inlet pressure at duty point flow in Fig. 10 is less than that for $80 \%$ flow but at 0.4 atm, it is more for both the models. The vapour volume fraction at $100 \%$ flow rate for $0.4 \mathrm{~atm}$ covers almost all the suction passage (from inlet to outlet). Asymmetry of extension of water vapour region is seen in $100 \%$ flow rates plots also but both SST and $\kappa-\varepsilon$ turbulence plots are similar in nature.

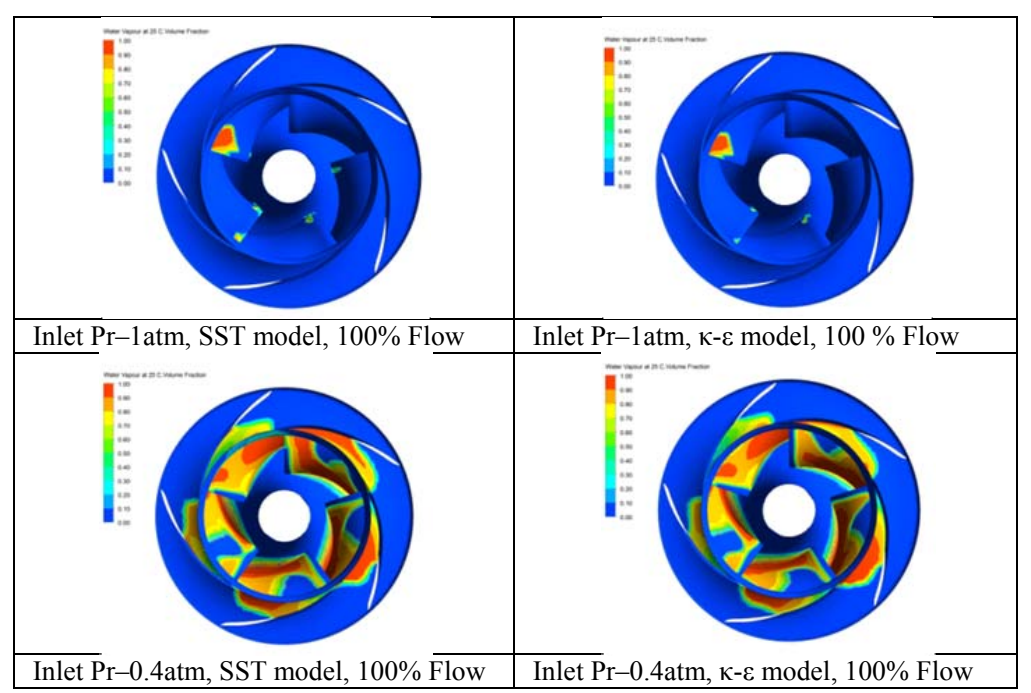

Fig. 10. Comparison of water vapour volume fraction at $100 \%$ of flow rate

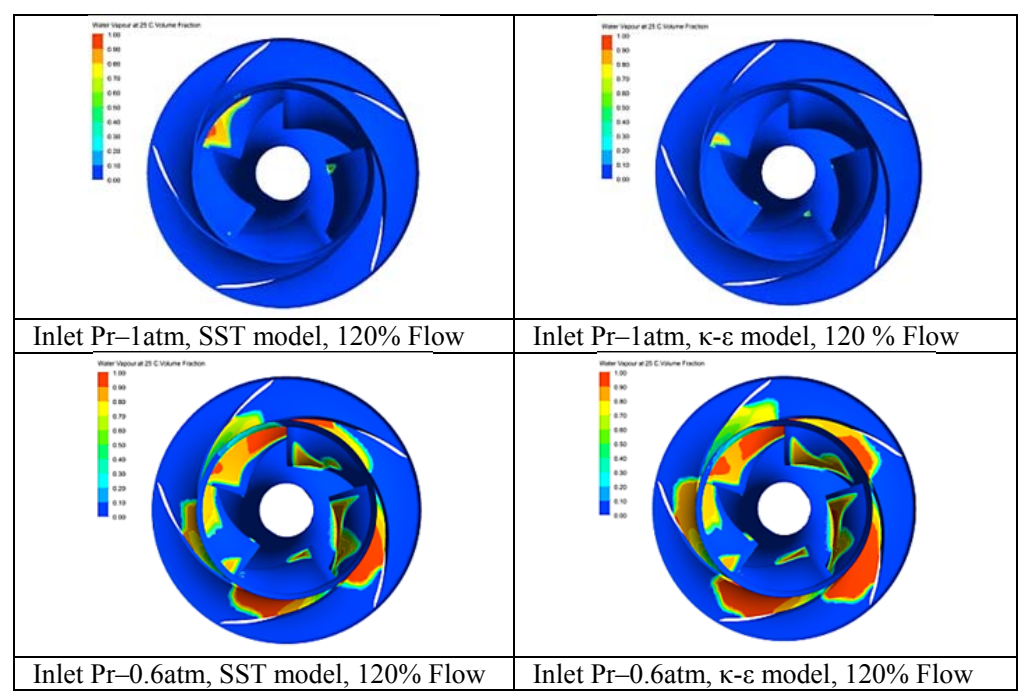

Fig. 11. Comparison of water vapour volume fraction at $120 \%$ of flow rate

The asymmetrical nature of cavitation zone may be due to presence of baffle plate in suction passage just at upstream of impeller eye. The purpose of placing this baffle plate is to guide the flow into impeller inlet with minimum losses. The flow enters into the rotating impeller after striking stationary baffle plate. There is local pressure drop due to presence of baffle, when flow passes through baffle plate which is clearly seen in Fig. 12. Since the simulation is conducted by applying frozen rotor interface condition between suction passage outlet and impeller inlet, the relative position is frozen and hence this drop of local pressure is visible in the contour plots.In reality, as impeller rotates, the relative position of vane changes with respect to baffle plate. Hence this pressure drop changes with respect to relative position of impeller vane to the baffle plate. 

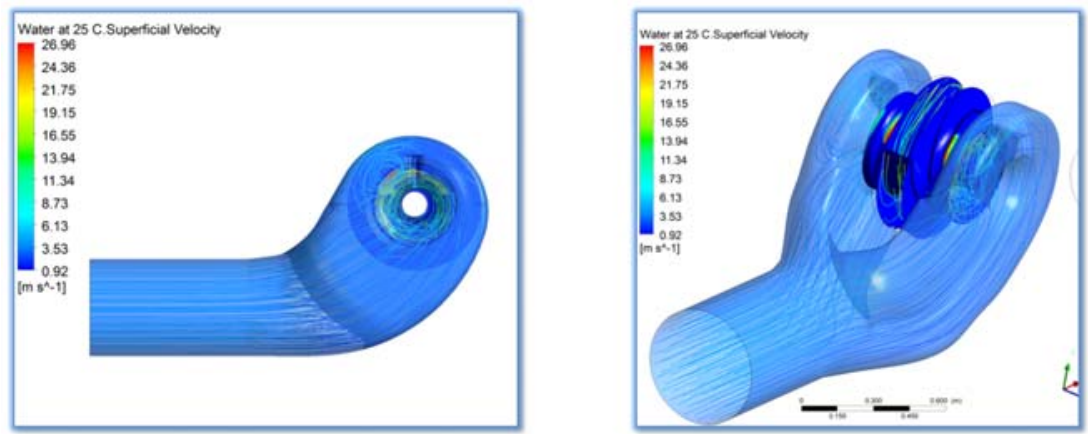

Fig.12- Streamline and contour plot in suction and impeller passage

\section{CONCLUSIONS}

The NPSHR value predicted from numerical simulations using SST model at duty point flow closely matches with experimental value. It is found to increase with increase in flow rate for both the SST and $\kappa-\varepsilon$ models. The inception of cavitation occurs early at part load condition. As the discharge increases, water vapour volume shifts towards outlet of runner. The development of cavitation zone on impeller is not uniform. The losses in different components are also found to vary with flow rates and turbulence models. The losses in impeller passage increase considerably due to cavitation while there is slight increase in losses in delivery casing. In case of suction, the losses are found to reduce due to bubbles formation in cavitation in some of the flow rates.

As the numerical simulation results with SST turbulence model have very good agreement with experimental values, therefore it is to be used for prediction of cavitation performance of the double suction pump using multiphase flow/cavitation analysis capabilities of commercially available software at design stage.

\section{NOMENCLATURE}

$\begin{array}{ll}\mathrm{H} & \text { head developed by pump, } \mathrm{m} \\ \mathrm{H}_{\mathrm{S}} & \text { headloss in suction, } \mathrm{m} \\ \mathrm{H}_{\mathrm{I}} & \text { headloss in impeller, } \mathrm{m} \\ \mathrm{H}_{\mathrm{C}} & \text { headloss in casing, } \mathrm{m} \\ \mathrm{TP}_{\mathrm{I}} & \text { total pressure at inlet, } \mathrm{Pa} \\ \mathrm{TP}_{\mathrm{O}} & \text { total pressure at delivery, } \mathrm{Pa} \\ \mathrm{P}_{\text {wvap }} & \text { water vapourpressure, } \mathrm{Pa} \\ \mathrm{g} & \text { acceleration due to gravity, } \mathrm{m} / \mathrm{s}^{2} \\ \rho & \text { liquid density, } \mathrm{kg} / \mathrm{m}^{3}\end{array}$

\section{ACKNOWLEDGMENT}

Authors would like to place on record their gratitude to the Management of Kirloskar Brothers Ltd. Pune, India for the encouraging attitude towards Research \& Engineering activities, which led to this paper. We are also very thankful to our colleagues in the Research \& Engineering Development, Pune for their co-operation during this work. Authors would also like to express its gratitude to MANIT, Bhopal for their support in carrying out this research work.

\section{REFERENCES}

[1] J. Karassik Igor, C. Krutzsch William, H. Fraser Warren and P. Messina Joseph, Pump Handbook, $2^{\text {nd }}$ Edition, McGraw-Hill International Editions, Ist Printing, 1986.

[2] Christopher E Brennen, An Introduction to Cavitation Fundamentals, Cavitation: Turbo-machinery\& Medical Applications, WIMRC FORUM 2011, 4th - 6th July, 2011, University of Warwick, UK.

[3] TAN Lei, ZHU BaoShan, CAO ShuLiang and WANG YuMing, "Cavitation flow simulation for a centrifugal pump at a low flow rate", Chinese science bulletin, Vol. 58, No. 8, pp.949-952, March 2013.

[4] S.G. Joshi A.S. Pujari, R.D. Kale and B.K. Sreedhar, "Cavitation studies on a model of primary sodium pump", Proceedings of FEDSM’02, 2005 ASME fluid engineering division summer meeting and exhibitions, June 19-23, 2005, Houston, TX, USA.

[5] Binama Maxime, Muhira Alex, Bisengimana Emmanuel, "Cavitation effects in centrifugal pumps-A review", International Journal of Engineering Research and Applications, Vol.6, Issue5, (Part-1), pp.52-63, May 2016.

[6] ANSYS - CFX users guide, V. 15.0, ANSYS Inc., USA, 2016.

[7] S. N. Shukla and Kshirsagar Jagadish, "Numerical prediction of cavitation in model pump", Proceedings of IMECE2008, 2008 ASME international Mechanical Engineering Congress and Exposition, Boston, Massachusetts, USA, November 2-5, 2008.

[8] Torbergsen Erik, Sigve Gjerstad, Hosoy Andreas and Aasland Brian, "Cavitation study in a high pressure water injection pump", Proceedings of the twentieth international pump user's symposium, 2003, p 49-55,.

[9] Gupta Amit and KshirsagarJT, "Numerical and experimental investigation of cavitation in a pump", Proceedings of FEDSM2005, The 2002 Joint US ASME European fluid engineering summer conference, July 14-18, 2002, Montreal, Canada

[10] Grist Edward, Cavitation and the Centrifugal Pump, Taylor \& Francis, 1998. 
[11] Hatano Shinya, Kang Donghyuk, Kagawa Shusaku, NohmiMotohiko and Yokota Kazuhiko," Study of Cavitation Instabilities in DoubleSuction Centrifugal Pump", International Journal of Fluid Machinery and Systems, Vol. 7, No. 3, July-September 2014, pp 94100

\section{AUTHOR PROFILE}

Shyam Narayan Shukla is a General Manager and in-charge, System Engineering at Corporate Research and engineering Development, Kirloskar Brothers Limited, Pune, India. His research interest is optimization of hydraulic design of pumps for improved performance. He is an extensive user of commercially available Computational Fluid Dynamics software and pursuing $\mathrm{PhD}$ in design of pumps. He has published papers in national and international indexed journals.

Ruchi Khare is Assistant Professor in Department of Civil Engineering, Maulana Azad National Institute of Technology (MANIT), Bhopal, India. Presently she is working in model testing of turbines and pumps, design optimization of hydraulic machines and rain water harvesting. She has supervised many PG theses and published papers in national and international indexed journals.

Vishnu Prasad is a Professor in Civil Engineering at Maulana Azad National Institute of Technology (MANIT), Bhopal, India. He is actively involved in model testing of turbines and pumps. His research interest is hydraulic machines and computational fluid dynamics. He has supervised $5 \mathrm{PhD}$ theses and $60 \mathrm{PG}$ theses and published papers in reputed national and international indexed journals. 